\title{
Proposal of Minicentrifuge-Compatible Centrifugal Microfluidic Device
}

\author{
Yoshiaki Ukita*, Yuichi Utsumi1 ${ }^{1}$ and Yuzuru Takamura ${ }^{2}$ \\ Department of Interdisciplinary Research, Graduate School of University of Yamanashi, \\ 4-3-11 Takeda, Kofu, Yamanashi 400-0016, Japan \\ ${ }^{1}$ Laboratory of Advanced Science and Technology for Industry (LASTI), University of Hyogo, \\ 3-1-2 Koto, Kamigori, Ako-gun, Hyogo 678-1205, Japan \\ ${ }^{2}$ School of Materials Science, Japan Advanced Institute of Science and Technology (JAIST), \\ 1-1 Asahi-dai, Nomi, Ishikawa 923-1211, Japan
}

(Received January 7, 2015; accepted January 15, 2015)

Key words: bioassay, enzymatic assay, immunoassay, microfluidics, centrifugal microfluidics, lab-on-a-chip, micro-TAS

In this paper, we report on the development of a mini-centrifuge-compatible microfluidic device that implements multistep liquid handling to realize bioassay protocols including enzymatic amplification steps such as enzyme-linked immunosorbent assay (ELISA). A device was developed by considering the geometry of the fluidic channel, the wettability of the microchannel, the volume and position of the chambers, and the entire assay process. The assay process includes injection of the sample into the reaction chamber, washing of the chamber, injection of the substrate, and injection of the stop buffer by two-mode (spin and stop) control that can be accomplished with an affordable minicentrifuge. During the assay protocol, both injection of the liquid into a chamber and draining of the liquid from the chamber are realized at a constant rotational speed, which is a key function for enzymatic amplification using a siphonshaped channel. The injected liquid is held in the reaction chamber, both in the presence and absence of centrifugal force, then the liquid is drained once a liquid exceeding the critical volume is injected into the chamber. The reaction chamber was prepared for enzymatic amplification. The device was then applied to assay the avidin-biotin system, and the resulting assay performance was demonstrated to be comparable to or better than the conventional titer plate assay performance.

\section{Background}

Microfluidic devices are attractive owing to the safe handling of high-risk samples, the handling of small volumes of samples in closed fluidic systems, and the ease of disposal of the fluid and the chip without first removing the fluid. However, some ${ }^{*}$ Corresponding author: e-mail: yukita@yamanashi.ac.jp 
commercial systems rely on complex, bulky, and expensive drivers consisting of syringe pumps and valves; thus, the widescale adoption of these systems is still not successful. ${ }^{(1,2)}$

Mark et al. have proposed the concept of microfluidic apps for easier promotion of microfluidic devices. ${ }^{(2)}$ In this concept, they propose the development of microfluidic devices that can be driven by off-the-shelf instruments so that various types of microfluidic devices can be driven like apps for smartphone devices. Moreover, users do not have to be trained in the operation of new instruments since the devices are driven by instruments with which the users are already familiar.

The most promising combination of drivers and devices is the centrifuge and centrifugal microfluidic devices. ${ }^{(3-9)}$ In the case of centrifugal microfluidic devices, the pumping of a small volume of liquid is realized using centrifugal force to apply pressure on the liquid so that pumping instruments (i.e., syringe pumps) and complex tubing networks are not necessary. From the viewpoint of widescale promotion of centrifugal pumping devices, the development of devices compatible with affordable and widely used centrifuges or minicentrifuges is ideal. On the other hand, complex assay protocols, such as enzyme-linked immunosorbent assay (ELISA), are not realized by simply rotating the device at a constant speed. Additionally, a typical minicentrifuge is only able to implement switching between two modes (stop and spin); therefore, existing centrifugal microfluidic devices are not compatible with the minicentrifuge.

The most simple centrifugal flow sequencing is realized by the rotatable cartridge concept. Kawai et al. integrated rotatable reagent cartridges on a rotating disk. In this device, the reagent to be injected into the reaction chamber is switched by the manual rotation of the cartridges. ${ }^{(9)}$ While this method requires the manual rotation of the cartridge, it seems to be compatible with the minicentrifuge, and an 1-lactate assay has been demonstrated. However, this assay protocol is relatively simple, with the addition of a reagent to the chamber, compared with a more complex protocol such as an ELISA, which also includes the draining of liquid from the reaction chamber. Furthermore, an ELISA requires not only the injection of the liquid into the reaction chamber but also a frequent draining of the liquid from the reaction chamber for the washing and enzymatic reaction steps. A technology capable of both high-order assay protocols and minicentrifuge compatibility has not yet been realized. In this paper, we report on the development of a centrifugal fluidic device capable of performing an ELISA, including the enzymatic reaction step, with two-mode (stop and spin) flow control as driven by a minicentrifuge.

\section{Experimental Methods}

\subsection{Reagents}

A thick negative photoresist (SU-8 3050) and an SU-8 developer were purchased from Nippon Kayaku Co., Ltd. Polydimethylsiloxane (PDMS, Silpot 184) was purchased from Dow Corning Toray Co., Ltd. Biotinylated bovine serum albumin (b-BSA) was purchased from Thermo Scientific Inc. and dissolved in ion-exchanged water to obtain a $2.0 \mathrm{mg} / \mathrm{ml}$ stock solution. Avidin was purchased from Sigma-Aldrich Co. LLC, and a 1.0 $\mathrm{mg} / \mathrm{ml}$ stock solution was prepared by dissolving in ion-exchanged water. Biotinylated horseradish peroxidase (HRP) was purchased from Thermo Scientific Inc., and a $5.0 \mathrm{mg} /$ 
$\mathrm{ml}$ stock solution was prepared. Avidin and biotinylated HRP stocks were mixed in a ratio of 1:1 (v:v) and incubated for over $24 \mathrm{~h}$ to obtain HRP-labeled avidin (HRP-avidin). 3,3',5,5' Tetramethylbenzidine (TMB) as a peroxidase substrate was purchased from Nacalai Tesque Inc. and prepared following the instructions of the manufacturer. Sulfuric acid was obtained from Wako Pure Chamicals Industries Ltd., and a $1 \mathrm{M}$ aqueous solution was prepared. Dulbecco's phosphate-buffered saline (DPBS) was prepared, and $\mathrm{pH}$ was adjusted to 7.4. Bovine serum albumin (BSA) was purchased from Sigma-Aldrich Co. LLC and dissolved to $1 \%(\mathrm{w} / \mathrm{v})$ in DPBS. The titer plate (Immuno Module) was purchased from Thermo Scientific Inc. Tween 20 was purchased from Nacalai Tesque Inc. and a $0.05 \%$ DPBS solution was prepared as a washing buffer.

\subsection{Centrifugal device}

Microchips made of PDMS, having a microchannel structure as shown in Fig. 1, were fabricated using standard soft lithography. ${ }^{(10)}$ A thick negative photoresist (SU-8 3050) was spin-coated onto a 4-inch silicon wafer to a $100 \mu \mathrm{m}$ thickness and soft-baked at $95{ }^{\circ} \mathrm{C}$. The microfluidic structure pattern was transferred onto the photoresist film by exposing UV light through a photomask having the fluidic pattern shown in Fig. 1. Then, the substrate was postbaked at $95{ }^{\circ} \mathrm{C}$, and the SU-8 relief pattern was developed to obtain a mold. The monomer and catalyst for the PDMS were mixed in a ratio of 10:1 and a PDMS film was cast on the mold with a 3-mm-thick spacer. A thin $(0.5-\mathrm{mm}$ thick) PDMS flat film was prepared on a plastic film in the same manner. These PDMS products were annealed at $75{ }^{\circ} \mathrm{C}$ for $40 \mathrm{~min}$, peeled from the mold and plastic film, and

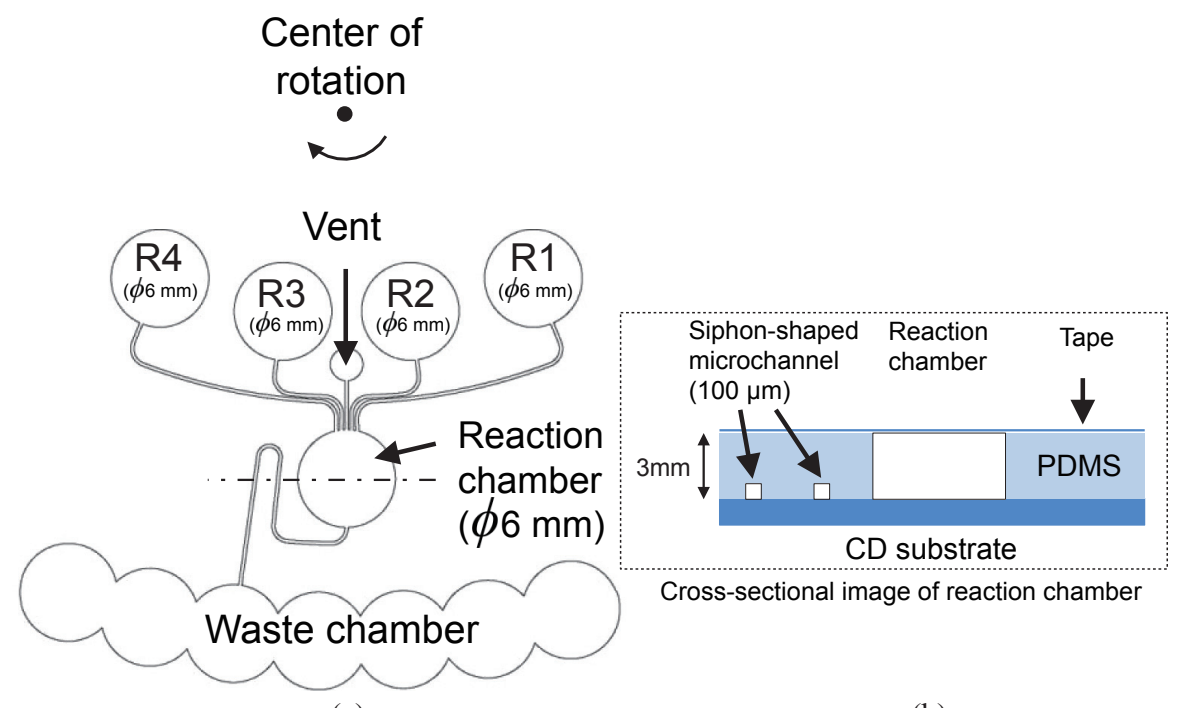

(a)

(b)

Fig. 1 (Color online) (a) Schematic illustration of a centrifugal device. Layout of the chambers and microchannels. (b) Cross-sectional view of the reaction chamber and siphon-shaped microchannel. 
then annealed at $200{ }^{\circ} \mathrm{C}$ for another $40 \mathrm{~min}$. Reaction chambers, waste chambers, and reagent stock reservoirs were formed by punching $6 \mathrm{~mm}$ holes through the 3-mm-thick PDMS fluidic chip. Air vent holes were punched with an $18 \mathrm{G}$ syringe needle. The punched PDMS chip was cleaned with ethanol and ion-exchanged water under ultrasonic conditions. After drying, the chip was bonded onto another thin PDMS film to cover the fluidic structure and to form a closed microchannel structure. The chip was then attached to a $12 \mathrm{~cm}$ compact disk (CD) substrate to obtain a centrifugal device. A laboratory-built PDMS titer plate was prepared for comparison with the centrifugal device and manual assay. To prepare the titer plate, a 5-mm-thick PDMS sheet was prepared with an array of $6 \mathrm{~mm}$ holes punched into the sheet and then cleaned as described. Finally, the PDMS sheet was bonded to a $0.5-\mathrm{mm}$-thick PDMS sheet.

\subsection{Device drivers and observation methods}

Two types of device drivers were used to drive the centrifugal devices. One was a servo motor driver with a stroboscope imaging system. ${ }^{(11)}$ Another was a commercially available minicentrifuge (Swing Man, ATT-101) using a homemade adapter for the attachment of the $\mathrm{CD}$ onto the minicentrifuge as shown in Fig. 2. The centrifugal device was attached to and screwed down on the rotational shaft. The acceleration of rotation was set to $500 \mathrm{rpm} / \mathrm{s}$ in the case of the servo motor driver. The standard stroboscope imaging method, which synchronizes a flash of strobe light with the rotation of the $\mathrm{CD}$, was utilized to obtain real-time images of the centrifugal fluidic pumping behavior. For detailed instructions, the methods can be found in the literature. ${ }^{(11)}$ The rotational speed was measured in advance while operating the minicentrifuge with an attached blank centrifugal device, and the speed position was marked at a rotational speed setting of $2000 \mathrm{rpm}$, with no other control support applied for this driver.

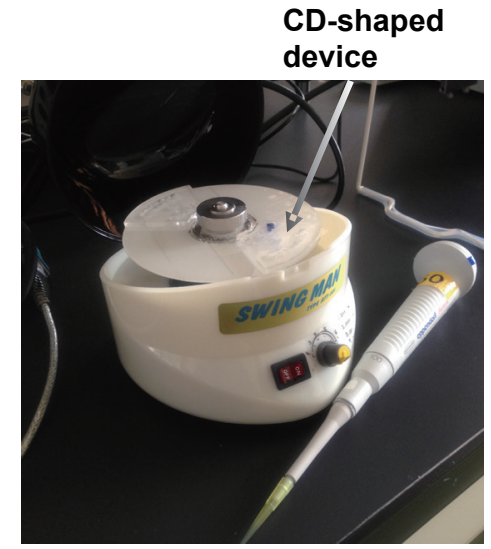

(a)

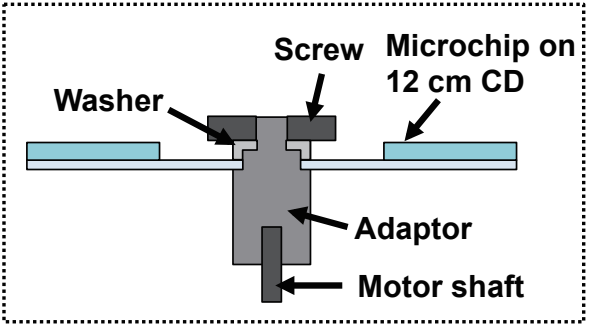

Cross-sectional image of device holder

(b)

Fig. 2. (Color online) (a) Minicentrifuge instrument attached to the centrifugal device. (b) Cross-sectional view of the device holder. 


\subsection{Solid-phase preparation for biotin-avidin interaction assay}

A b-BSA solution was prepared in DPBS at $20 \mu \mathrm{g} / \mathrm{ml}$ and $50 \mu \mathrm{l}$ of this solution was applied in the reaction chamber and incubated overnight at $4{ }^{\circ} \mathrm{C}$. The reaction chamber was washed three times with washing buffer followed by blocking with a $1 \%$ BSA solution in DPBS overnight at $4{ }^{\circ} \mathrm{C}$. The BSA solution was removed from the reaction chamber just before the assay and the chamber was washed three times with washing buffer. The reagent stock reservoirs, reaction chamber, and waste chamber were covered with adhesive tape. While the reaction chamber was covered completely, the reagent stock reservoirs and waste chamber were sealed with a small gap at the top of the chamber for access by pipette tips and venting of air. As shown in the supporting material, the hydrophilic postmodification of the PDMS surface was effective to obtain a higher signal; however, it resulted in more difficulty in controlling the assay. Therefore, no post-modification of PDMS, such as exposure to oxygen plasma, was applied in this experiment.

\subsection{Assay operation}

A sample of HRP-avidin was prepared in a $1 \%$ BSA solution with a concentration of 0.05 $\mathrm{ng} / \mathrm{ml}$ to $5000 \mathrm{ng} / \mathrm{ml}$. $50 \mu \mathrm{l}$ of the sample was applied to the reagent stock chamber 1 (R1), and the device was spun at $2000 \mathrm{rpm}$ and then incubated for $30 \mathrm{~min}$ at room temperature. $50 \mu \mathrm{l}$ of washing buffer was applied to reagent stock chamber 2 (R2), and the device was spun at $2000 \mathrm{rpm}$. This operation was repeated five times. $30 \mu \mathrm{l}$ of the TMB substrate was applied to reagent stock chamber 3 (R3), and the device was spun at $2000 \mathrm{rpm}$ followed by $30 \mathrm{~min}$ of incubation at room temperature. $20 \mu \mathrm{l}$ of $1 \mathrm{M} \mathrm{H}_{2} \mathrm{SO}_{4}$ was then injected into reagent stock chamber 4 (R4), and the device was spun at $2000 \mathrm{rpm}$. The liquid stock in the reaction chamber was transferred to the titer plate for analysis. The optical density (OD) at $450 \mathrm{~nm}$ was measured and a calibration curve was prepared.

For comparison with the centrifugal assay, a conventional, manual-style assay using a laboratory-built PDMS titer plate was conducted. The solid phase was prepared by following the same manner of preparation of the centrifugal device. Samples were prepared in $1 \%$ BSA solutions, and $50 \mu$ of the sample was applied to the titer plate and incubated for $30 \mathrm{~min}$ at room temperature. Then, each reaction chamber was washed with $100 \mu \mathrm{l}$ of washing buffer three times. $30 \mu \mathrm{l}$ of TMB was then applied to the reaction chambers and incubated for $30 \mathrm{~min}$ at room temperature. Next, $20 \mu \mathrm{l}$ of $1 \mathrm{M} \mathrm{H}_{2} \mathrm{SO}_{4}$ was applied to the reaction chambers to stop the color development. Finally, the liquids were transferred to the titer plate to read the OD at $450 \mathrm{~nm}$ and a calibration curve was prepared.

\section{Results and Discussion}

\subsection{Autonomous draining of liquids under constant rotational speeds}

The observed fluidic behaviors are shown in Figs. 3 and 4. In the first liquid pumping, $50 \mu \mathrm{l}$ of dyed water was injected into R1 and the device was spun at 2000 $\mathrm{rpm}$. The water pumped by the generated centrifugal force flowed out from R1 and was transferred to the downstream reaction chamber as shown in Figs. 3(a) and 3(b). The 

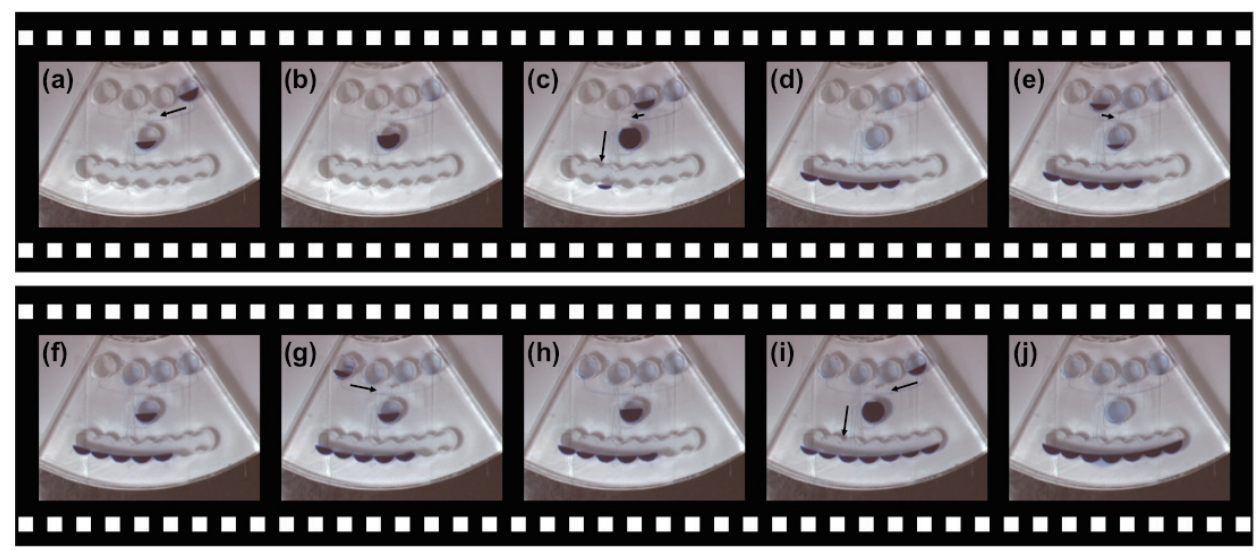

Fig. 3. (Color online) Real-time observation of fluidic behavior controlled by the servo motor driver.

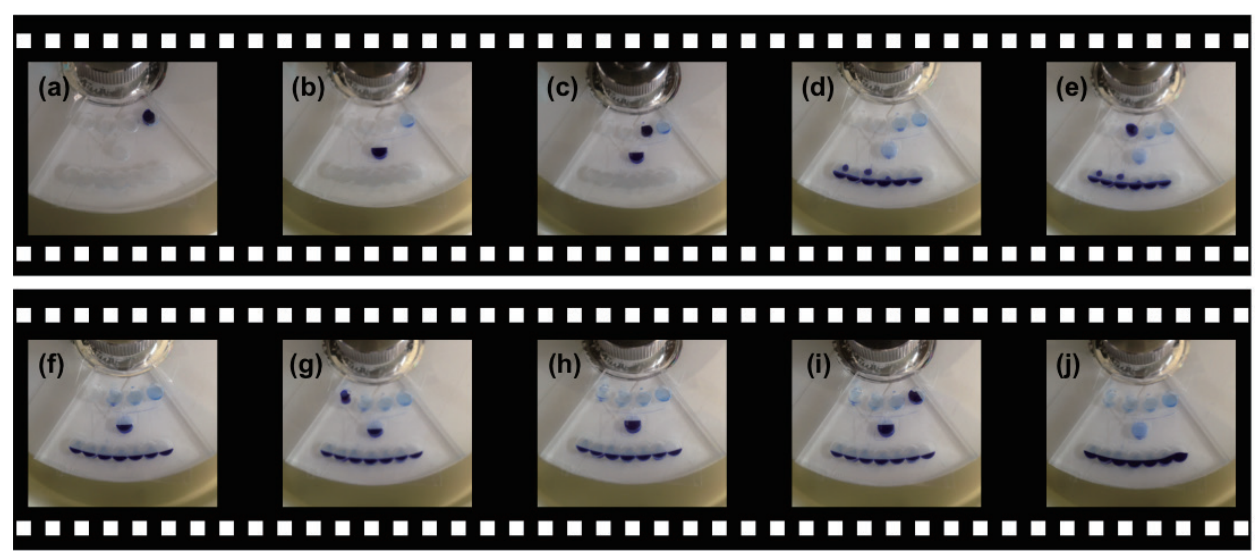

Fig. 4. (Color online) Fluidic behaviors observed before and after centrifugal pumping by minicentrifuge instrument.

transferred water was held in the reaction chamber both in the presence and absence of centrifugal force because of the siphon structure connected to the bottom of the chamber, as shown in Fig. 3(c). Another $50 \mu \mathrm{l}$ of dyed water was injected into R2 and the device was spun at $2000 \mathrm{rpm}$ again to transfer the water to the reaction chamber. Owing to the increase in water head pressure in the reaction chamber, the water meniscus in the siphon increases in the microchannel. Once the meniscus reached the top of the siphon and turned the U-shaped corner of the channel, the water drained from the reaction chamber, as shown in Fig. 3(c). Afterward, the liquid was continually pumped, first emptying R2 
followed by the reaction chamber as shown in Fig. 3(d). Therefore, it was successfully demonstrated that the autonomous draining of the liquid from the reaction chamber was implemented without applying external triggers such as changing the rotational speed ${ }^{(5,7,8)}$ or laser irradiation. (6) $30 \mu \mathrm{l}$ of the water sample was applied to R3 and the water was transferred to the reaction chamber by spinning the device at $2000 \mathrm{rpm}$. The transferred liquid was successfully held in the reaction chamber as shown in Figs. 3(e) and 3(f). 20 $\mu \mathrm{l}$ of the water sample was injected into R4 and the device was again spun at $2000 \mathrm{rpm}$. Because the total liquid volume in the reaction chamber was still less than the critical volume (approximately $80 \mu \mathrm{l}$ ) to initiate draining, the liquid was held inside the reaction chamber as shown in Figs. 3(g) and 3(h). This reveals that the addition of the chemical required to stop the enzymatic reaction can be implemented by this method. Finally, $50 \mu \mathrm{l}$ of the water sample was injected into R1 again, and the device was spun at 2000 rpm to transfer the sample into the reaction chamber. After reaching critical volume, the draining of liquid from the reaction chamber was triggered again and the same draining behavior was observed as shown in Figs. 3(i) and 3(j). This reveals that the liquid transportation can be repeated even if the microchannel previously experienced the flow. Moreover, we confirmed the same flow behavior using the washing buffer, suggesting that the device is not sensitive to the small difference in the physical property of the liquid. We would like to specify that it is important to use a hydrophobic material to demonstrate the concept because it is important for preventing the capillary action in the siphon during the rest state of the device. While it is often necessary to modify the surface of the microchannel to be hydrophilic by oxygen plasma treatment of the PDMS, it is not recommended in this setup because the liquid flows in the siphon when the device is at rest, and the liquid is transported to a waste reservoir during the next spinning even if the total volume is below the critical volume. PDMS hydrophilicity is not stable for long durations; therefore, the concept proposed here is advantageous from the viewpoint of the PDMS stability. A method exists for using a siphon structure for the valving and transportation of liquid on a centrifugal device; ${ }^{(3)}$ however, the proposed concept is different from this concept because of the triggering principle. In the case of siphon valving, the liquid is drawn into the hydrophilic siphon microchannel using capillary action. Therefore, it is not effective for this study because liquid is drawn into the siphon microchannel when the device is at rest. We demonstrated the device control using a minicentrifuge instrument. The conditions of the liquid before and after device spinning are shown in Fig. 4. From these results, we conclude that there is no difference between the minicentrifuge driver and the servo driver experiment. Therefore, the device can be driven with a simple driver lacking precise rotational control.

\subsection{Avidin-biotin interaction assay}

Figure 5 depicts the observed flow behavior during the avidin-biotin interaction assay and corresponding reaction. When the sample was applied to the reaction chamber, the HRP-avidin reacts with b-BSA as shown in Figs. 5(a) and 5(b). To wash unbound (free) HRP-avidin, $50 \mu \mathrm{l}$ of the washing buffer was injected into R2 five times, and the draining of the liquid from the reaction chamber was repeated three times during these steps. In the case of first washing, the total volume of the washing buffer and sample was $100 \mu$, 
(a)
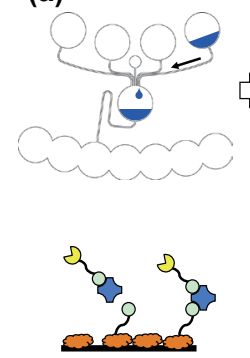

(e)

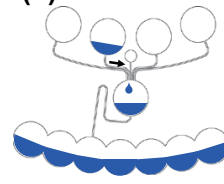

口

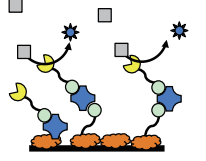

(b)
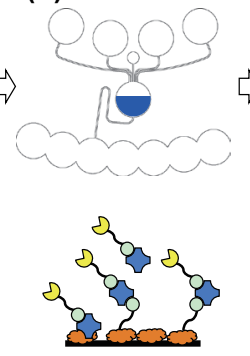

(f)

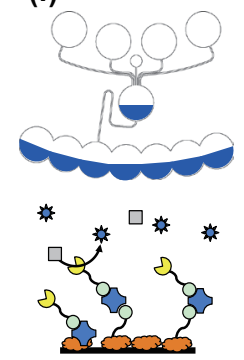

(c)
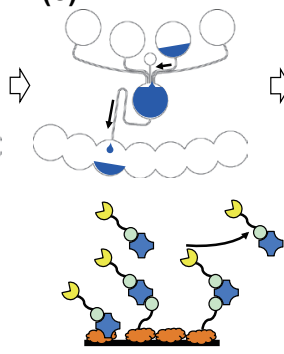

(d)

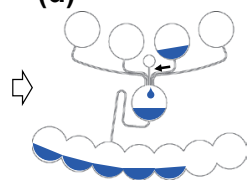

(g)

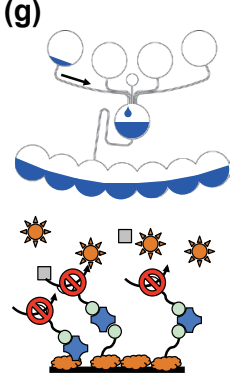

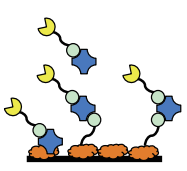

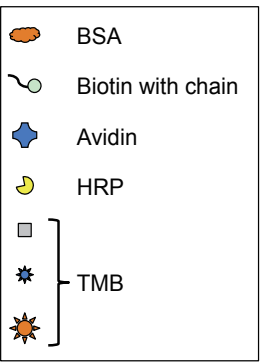

Fig. 5. (Color online) Depicted fluidic behavior and corresponding reaction scheme during avidin-biotin interaction assay.

and the mixture was drained via the siphon with the reaction chamber emptying as shown in Fig. 5(c). For the second washing buffer pumping, the washing buffer was injected into the empty reaction chamber and the buffer was held inside the reaction chamber as shown in Fig. 5(d). By repeating these steps, the filling of the washing buffer and draining were repeated three times. Afterward, $30 \mu \mathrm{l}$ of the TMB substrate was pumped into the empty reaction chamber and the color was developed according to the enzymatic reaction, as shown in Figs. 5(e) and 5(f). Finally, $20 \mu \mathrm{l}$ of $1 \mathrm{M} \mathrm{H}_{2} \mathrm{SO}_{4}$ was added to stop the enzymatic reaction, while the mixture remained in the reaction chamber as the total volume was below the critical volume.

Figure 6 shows the obtained calibration curve for the avidin-biotin interaction assay. In the case of the centrifugal device, all data were measured by independent assay, while in the case of the manual assay, the data were measured in one experiment. The calibration curve demonstrates a clear response to the concentration of HRP-avidin and no significant error is found in the centrifugal device. Moreover, we confirmed a significant difference between specific and nonspecific signals, indicating that the detection system has sufficient specificity, as shown in Fig. S2. It is proven that washing in the centrifugal device is comparably effective because the calibration curve exhibits a similar trend to the manual assay, suggesting effective removal of free HRP from the reaction chamber during the washing steps. 


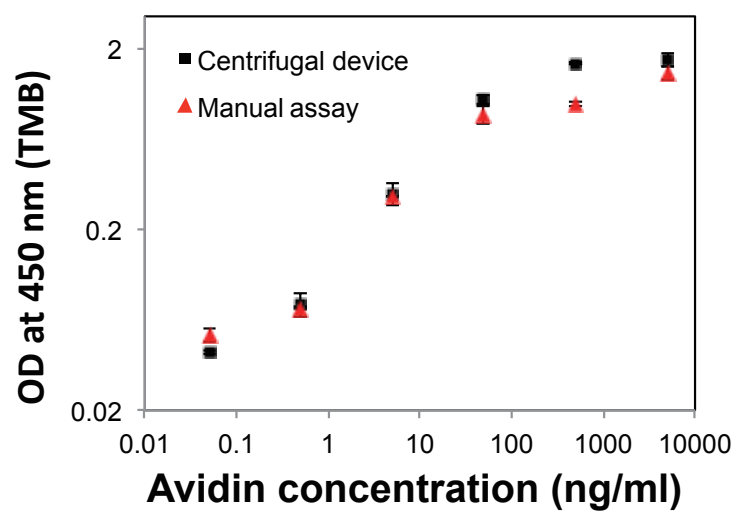

Fig. 6. (Color online) Calibration curve of avidin-HRP obtained by centrifugal device and manual assay.

Generally, it is essential to remove the fluid from the reaction chamber between steps of the protocol. In the case of the conventional titer plate assay, the liquid in the reaction chamber was removed by aspirating and tapping to blot on a paper towel. These operations increase the risk of contamination of the reaction chamber, thus increasing the potential danger when the sample is clinically hazardous. On the other hand, in the case of the centrifugal device, the liquid is pumped by centrifugal force and drained into the waste chamber located downstream of the reaction chamber. Here, the liquid does not need to be removed from the device, increasing the safety of the system.

\section{Conclusions}

The concept of a centrifugal microfluidic device compatible with a minicentrifuge instrument is proposed. It is important to realize the complexity of fluidic control under two-mode (stop and spin) control to drive the centrifugal device with many minicentrifuge instruments. To realize this concept, the autonomous draining of liquid and the complex assay protocol, including multistep washing and enzymatic amplification, are demonstrated without applying an external trigger, but with an internal trigger of constant rotation. The latter can be operated through the use of a standard, commonly used minicentrifuge. The device assay performance is comparable to or better than the conventional titer plate assay performance, and it is safer because the reagents injected in the device do not have to be removed.

\section{Acknowledgements}

The authors appreciate the financial support provided by Strategic Information and Communications R\&D Promotion Programme (SCOPE) of the Japanese Ministry of Internal Affairs and Communications. 


\section{References}

1 D. Mark, S. Haeberle, G. Roth, F. Stetten and R. Zengerle: Chem. Soc. Rev. 39 (2010) 1153.

2 D. Mark, F. Stettena and R. Zengerle: Lab Chip 12 (2012) 2464.

3 D. C. Duffy, H. L. Gillis, J. Lin, N. F. Sheppard, Jr. and G. J. Kellogg: Anal. Chem. 71 (1999) 4669.

4 R. Gorkin, J. Park, J. Siegrist, M. Amasia, B. S. Lee, J.-M. Park, J. Kim, H. Kim, M. Madou and Y.-K. Cho: Lab Chip 10 (2010) 1758.

5 S. Lay, S. Wang, J. Luo, L. J. Lee, S. T. Yang and M. J. Madou: Anal. Chem. 76 (2004) 1832.

6 B. S. Lee, J.-N. Lee, J-M Park, J.-G. Lee, S. Kim, Y.-K. Cho and C. Ko: Lab Chip 9 (2009) 1548.

7 Y. Ukita, S. Kondo, T. Azeta, M. Ishizawa, C. Kataoka, M. Takeo and Y. Utsumi: Sens. Actuators, B 166-167 (2012) 898.

8 J. Siegrist, R. Gorkin, L. Clime, E. Roy, R. Peytavi, H. Kido, M. Bergeron,T. Veres and M. Madou: Microfluid. Nanofluid. 9 (2010) 55.

9 T. Kawai, N. Naruishi, H. Nagai, Y. Tanaka, Y. Hagihara and Y. Yoshida: Anal. Chem. 85 (2013) 6587.

10 Y. Ukita and Y. Takamura: Microfluid. Nanofluid. 15 (2013) 829.

11 D. C. Duffy, J. C. McDonald, O. J. A. Schueller and G. M. Whitesides: Anal. Chem. 70 (1998) 4974.

12 Y. Ukita and Y. Takamura: Microfluid. Nanofluid. (2014) DOI 10.1007/s10404-014-1426-7.

\section{Appendix}

\section{Investigation of solid phase}

The optimization of the PDMS surface for the avidin-biotin interaction assay was carried out. To prepare the PDMS titer plate, a 5-mm-thick PDMS sheet was punched with a $6 \mathrm{~mm}$ puncher. For the comparison of hydrophilic and hydrophobic surfaces, two types of PDMS titer plates with and without air plasma treatment were prepared. The solid-phase preparation was carried out by the method described in the experimental part. For the assay, HRP-avidin was diluted 105 times by 1\% BSA solution, and $50 \mu \mathrm{l}$ of the liquid was applied in the reaction chambers, and incubated for $30 \mathrm{~min}$ at $37^{\circ} \mathrm{C}$. The reaction chamber was washed three times by washing buffer. Then, $50 \mu \mathrm{l}$ of TMB was applied and incubated for $30 \mathrm{~min}$ at $37^{\circ} \mathrm{C}$. Finally, $50 \mu \mathrm{l}$ of $1 \mathrm{M} \mathrm{H}_{2} \mathrm{SO}_{4}$ was applied and the optical density at $450 \mathrm{~nm}$ was measured by the plate reader. Figure S1 shows the results of six independent experiments. While the polystyrene titer plate and air plasma-treated PDMS typically exhibit a higher signal than the PDMS titer plate without air plasma treatment, the nonspecific signal biotin (-) of the PDMS without air plasma treatment is more stable and lower than others.

\section{Specificity of avidin-biotin assay system}

In this assay system, the nonspecific signal (NSS) due to nonspecific binding of HRPavidin onto the solid surface was expected to exhibit the same trend as that exhibited by the specific signal (SS). To confirm that the SS is reasonably higher than the NSS in the 

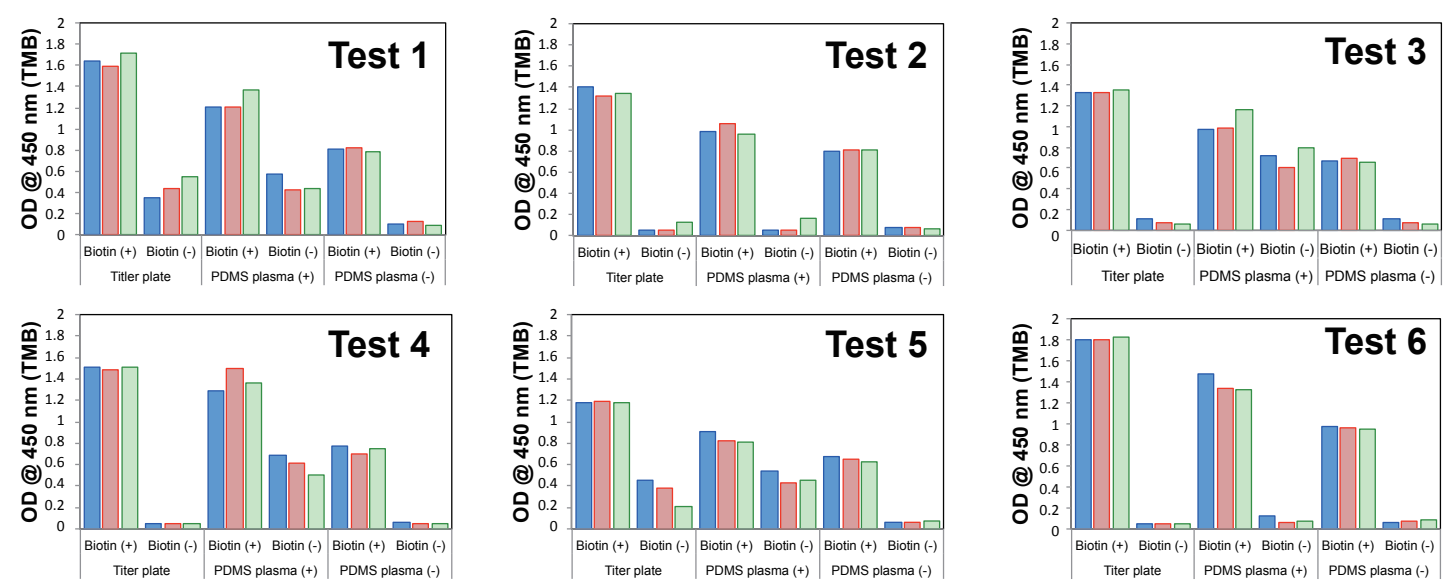

Fig. S1. (Color online) Comparison of specific signal [biotin $(+)$ ] and nonspecific signal [biotin $(-)$ ] obtained by titer plates. Results of six independent tests with $n=3$. All measurements were carried out at an avidin concentration of $5 \mathrm{ng} / \mathrm{ml}$.

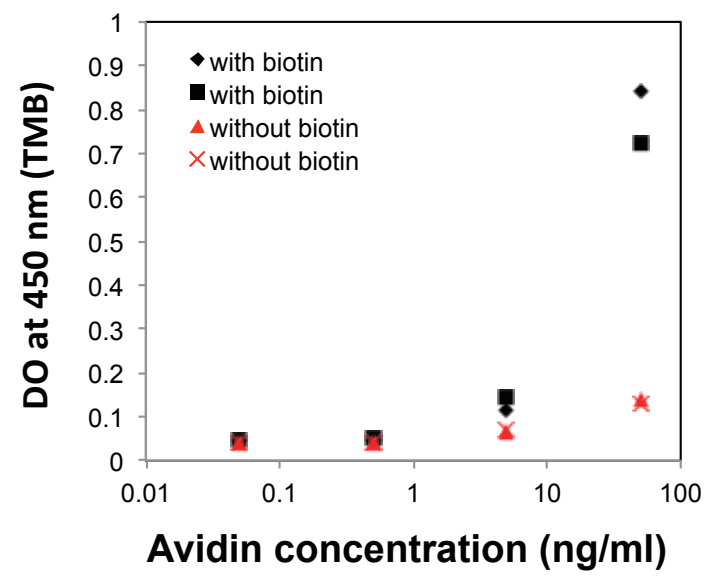

Fig. S2. (Color online) SS and NSS of the avidin-biotin interaction assay system.

assay, we compared the systems using solid surfaces with and without biotinylated BSA. Biotinylated BSA was adsorbed on the surface of the PDMS titer plate and the assay was carried out manually. The solid phase without immobilization of biotinylated BSA was prepared to estimate the NSS, while PBS(-) was applied during biotinylated BSA immobilization. For the assay, HRP-avidin was prepared in 1\% BSA solution, and $50 \mu \mathrm{l}$ of each sample was applied in the reaction chambers and incubated for $30 \mathrm{~min}$ at $37^{\circ} \mathrm{C}$. 
The reaction chamber was washed three times by washing buffer. Then, $50 \mu \mathrm{l}$ of TMB was applied and incubated for $30 \mathrm{~min}$ at $37^{\circ} \mathrm{C}$. Finally, $50 \mu \mathrm{l}$ of $1 \mathrm{M} \mathrm{H}_{2} \mathrm{SO}_{4}$ was applied and the optical density at $450 \mathrm{~nm}$ was measured by the plate reader. The result is shown in Fig. S2, and a clear difference between the SS and the NSS is confirmed; therefore, it is concluded that this assay system detects the specific interaction of avidin and biotin. 